\title{
Strates
}

STRATES Matériaux pour la recherche en sciences sociales

Hors-série | 2002

Parcours dans la recherche urbaine, Michel Rochefort, un géographe engagé

\section{Quarante ans de relations avec le Brésil}

\section{Maria Adélia Aparecida De Souza}

\section{(2) OpenEdition \\ Journals}

Édition électronique

URL : http://journals.openedition.org/strates/561

DOI : $10.4000 /$ strates.561

ISSN : 1777-5442

Éditeur

Laboratoire Ladyss

Édition imprimée

Date de publication : 1 janvier 2002

ISSN : 0768-8067

Référence électronique

Maria Adélia Aparecida De Souza, «Quarante ans de relations avec le Brésil », Strates [En ligne], Horssérie | 2002, mis en ligne le 18 mai 2005, consulté le 08 septembre 2020. URL : http://

journals.openedition.org/strates/561 ; DOI : https://doi.org/10.4000/strates.561

Ce document a été généré automatiquement le 8 septembre 2020

Tous droits réservés 


\title{
Quarante ans de relations avec le Brésil
}

\author{
Maria Adélia Aparecida De Souza
}

$1 \quad$ Michel Rochefort découvre le Brésil en 1956, lors d'un séjour de sept mois pendant lequel il participe au Congrès international de géographie de Rio de Janeiro, prépare sa thèse complémentaire de doctorat d'État sur "Pluviosité et écoulement dans deux bassins fluviaux brésiliens", et prend connaissance de l'Amazonie, du Nord-Est et du Sud du pays, grâce à l'aviation militaire brésilienne. Il est également invité par Milton Santos pour une conférence à l'université de Salvador. À la suite, il revient au Brésil pour près de deux ans, en 1960-1961, enseigner à l'université fédérale de Pernambuco (Recife), puis collaborer aux recherches du Conseil national de géographie (IBGE) à Rio de Janeiro et du centre de recherche de Salvador. Durant toute cette première période, son rôle repose essentiellement sur la diffusion au Brésil des idées et des méthodes de recherche de la géographie française.

2 À partir de 1966, une nouvelle collaboration s'instaure entre Michel Rochefort et divers services d'aménagement urbain ou régional qui commencent à se créer au Brésil: SERFAU à Rio de Janeiro, Groupe de décentralisation industrielle à São Paulo, puis, de 1974 à 1978, de façon plus constante, Commission Nationale de Politique Urbaine (CNPU) à Brasilia et secrétariat à l'Aménagement du territoire de l'État de São Paulo. Durant cette longue période, il assure en même temps des cours et conférences dans de très nombreuses universités brésiliennes: Porto Alegre, Florianapolis, Curitiba, Presidente Prudente, São Paulo, Brasilia, Salvador, Recife, Fortaleza, Manaus, etc.

3 Après 1980, commence une troisième phase de la collaboration entre Michel Rochefort et les géographes brésiliens. Face à l'essor rapide et profond de la géographie brésilienne, il n'est plus question d'enseigner à la lumière de la géographie française mais de participer à des échanges d'idées et de méthodes, soit dans le cadre de programmes de recherches franco-brésiliens, soit dans le cadre de congrès, colloques, ou journées de travail. Dans cette nouvelle optique, ce furent d'abord divers séjours entre 1980 et 1989, puis après une interruption de plusieurs années, une reprise des échanges à partir de 1997, le point culminant ayant été sa participation au colloque que j'ai organisé à São Paulo en hommage à cette longue collaboration, en mars 1998, dont je 
présente ici un résumé des débats. Il s'y est ajouté la publication en portugais d'un livre de textes choisis de son cuvre. ${ }^{1}$

Un témoignage global : le colloque de São Paulo « Réseaux et systèmes - réflexions sur l'urbain et l'urbanisation "

4 Michel Rochefort est devenu un personnage important pour la géographie brésilienne, tant par ses activités universitaires que par le fait qu'il nous a aidé à connaître notre pays et notre territoire, et tout spécialement les processus d'urbanisation et de régionalisation. Ceci a permis de produire de nombreux textes auprès des institutions universitaires et de la Fondation de l'Institut brésilien de géographie et statistique, depuis les années soixante.

5 Pour l'université brésilienne, sa collaboration a été inestimable, puisqu'au moment où nous avons organisé notre troisième cycle, il nous a aidé en formant des dizaines de docteurs et maitres qui ont ensuite assumé les cours, non seulement en géographie, mais aussi en économie, architecture, urbanisme et génie civil. On ne peut donner ici la liste de ses nombreux élèves brésiliens, mais le respect pour Michel Rochefort a été largement vérifié par le nombre et les provenances des représentants des universités nationales et étrangères qui sont venus à São Paulo : ceux de São Paulo, bien sûr, mais aussi ceux de Rio de Janeiro, Bahia, Pernambuco, Ceara, Mato Grosso do Sul, Amazonas, Brasilia, Londrina, Uberlandia, Sorocaba, auxquels se sont ajoutés des élèves et collègues de l'étranger : de France, du Chili, d'Argentine.

6 Tous étaient là pour l'hommage à un maître et à un ami. Mais ce qui est important ici, c'est de donner au lecteur une idée de l'amplitude et du contenu de cette réunion universitaire.

7 Tout d'abord il faut dire que le thème du colloque « Réseaux et systèmes » a été choisi avec un double objectif: le premier était de pouvoir rendre compte de l'œuvre de Michel Rochefort, qui, avec sa thèse de doctorat sur le Réseau urbain de l'Alsace ${ }^{2}$, a introduit ce concept dans la géographie contemporaine; le second était d'essayer d'actualiser la réflexion sur réseaux et systèmes au regard des nouvelles approches rencontrées dans la géographie brésilienne et dans la géographie et les sciences humaines en général.

8 Ces objectifs ont été pleinement atteints : diverses communications ont présenté, soit des recherches dérivées de la méthodologie proposée par Rochefort, soit les résultats d'investigations géographiques d'avant-garde sur les concepts de réseaux et systèmes, devant plus de deux cents participants. Plusieurs conférences que je ne peux qu'évoquer ici ont également laissé de précieux enseignements, soit sur la thématique de la réunion et l'évolution de la pensée géographique brésilienne, soit sur la personnalité de Michel Rochefort et sa générosité dans des moments politiques aigus pour l'Amérique Latine.

9 Jacques Chonchol a apporté à propos de l'Amérique Latine une très intéressante contribution sur le processus de globalisation qui sera publiée par la revue de l'Institut d'études avancées de l'université de São Paulo.

Pedro Geiger, ancien collègue de Michel et Regina Rochefort à l'IBGE (Institut brésilien de géographie et statistique à Rio de Janeiro), a fait une conférence pleine d'émotion et de beaux souvenirs intitulée Réseaux urbains hier et aujourd'hui, dans laquelle il a mis en parallèle l'influence de Michel Rochefort dans la géographie urbaine et régionale brésilienne et l'histoire de l'IBGE. Il l'a terminée, faisant en cela appel au poète, par une 
phrase très révélatrice de l'ambiance du colloque : «Voyageur, les routes n'existent pas... les routes, on les fait en marchant... ».

11 Manuel Correia De Andrade qui a coordonné une table ronde sur Les villes dans les pays sous-développés, nous a fait parcourir tout le chemin des études sur ce thème. Rappelant le premier séjour de Michel Rochefort à Recife et tout ce qu'il a laissé comme héritage scientifique jusqu'à aujourd'hui, il a également fait état de la façon dont celui-ci l'avait soutenu, à la fois comme collègue et comme ami, lors de son séjour en France au moment de son exil politique pendant les premières années de la dictature militaire brésilienne. Hélène Lamicq, dans sa conférence de clôture, s'est livrée à une analyse de son œuvre, pleine de critique et d'élégance, tout en parcourant la vie du professeur et en évoquant l'exceptionnel être humain qu'il est.

Quant à Michel Rochefort, il a ouvert le colloque. C'est sur sa conférence inaugurale que je m'attarderai ici.

L'aménagement du territoire en France et le nouveau rôle de la Datar

Il s'agit d'une réflexion critique et auto-critique sur l'aménagement du territoire français et son propre rôle dans ce processus, sur l'importance et les limites des notions de réseau et de système dans l'orientation de la planification territoriale française depuis cinquante ans.

a drançaise s'est développée à l'issue de la deuxième guerre mondiale, dans une ambiance de développement industriel et de croissance rapide du produit intérieur brut. Il a ensuite expliqué pourquoi les notions de réseaux et de systèmes urbains ont été retenues et sont devenues les jalons de l'aménagement territorial français et pour cela il a choisi de retracer l'histoire du programme des métropoles d'équilibre.

Après 1974, ces ambitions pour modifier l'organisation de l'espace français ont été partiellement abandonnées. Pourquoi cet abandon? Est-ce à cause de la crise économique et de ses effets? ou bien est-ce parce que ces programmes étaient fondés sur des concepts erronés ? De toute façon, à partir de 1974, l'aménagement du territoire en France sera cantonné à la résolution des problèmes immédiats. Il faut attendre les années quatre-vingt-dix pour qu'apparaisse une nouvelle réflexion, à la recherche de nouvelles bases pour un aménagement territorial français mieux adapté à l'évolution de la société et de l'économie. C'est dans un service de la Datar, nommé Prospective et Territoires qu'on peut trouver les bases de ces analyses.

Mais avant cela, il faut revenir à l'après-guerre et au début de la planification française, pour voir le rôle de la notion de réseaux de villes dans le montage d'un programme d'aménagement. Cet aménagement du territoire est né dans un service du Commissariat général au Plan. Ce dernier était chargé du développement économique de la France, et sa première tâche était d'organiser un système de subventions pour chaque secteur de l'économie, afin de faciliter la réanimation du capitalisme français après les destructions de la guerre.

C'est là que commencent les "Années glorieuses ", comme les appellent les français : trente années de croissance économique jusqu'en 1974, avec une croissance annuelle du PIB aux environs de $5 \%$ à $6 \%$. Dans ce contexte, le Commissariat général au Plan, qui auparavant ne s'occupait que de l'économie, commença aussi à se préoccuper de l'organisation de l'espace, et à inaugurer l'aménagement du territoire. Dans les années cinquante, il crée la Commission Nationale pour l'Aménagement du Territoire, la CNAT, 
dont l'un des cinq groupes fut chargé de la planification de l'armature urbaine française. À ce moment là, le livre de Jean-François Gravier Paris et le désert français ${ }^{3}$ attirait beaucoup l'attention. À cause du développement industriel, Paris avait une croissance plus rapide que les autres villes françaises. Il fallait donc s'occuper de la relocalisation de l'industrie et essayer de diminuer les effets de la concentration parisienne par rapport au développement des autres régions.

Le premier programme dans ce sens fut celui de la décentralisation industrielle aux environs de 1956, avec peu de bases territoriales pour l'organiser, puisque ce service était dans les mains des ingénieurs des grandes écoles qui n'avaient pas une connaissance approfondie de la géographie de la France. Cette décentralisation a été faite en outre sans prendre en considération le problème des villes.

Par la suite, le Commissariat au Plan a compris qu'il ne fallait pas seulement s'occuper de la décentralisation industrielle, mais aussi du système des villes qui devraient devenir les pôles de cette décentralisation et servir à d'autres stratégies de développement régional. Dans l'optique d'un accroissement économique rapide, cet aménagement du système des villes devait permettre une croissance harmonieuse entre les régions françaises. Il fallait corriger les déséquilibres régionaux et arriver à une bonne maîtrise de chaque région avec une bonne croissance, un bon réseau urbain et un bon pôle principal pour chacune d'elles.

Quelques géographes avaient à l'époque lancé l'idée des réseaux régionaux de villes souvent à partir de thèses orientées par Pierre George, dont celle de Michel Rochefort soutenue en 1958, et, pour diverses raisons, celui-ci a été invité à diriger les recherches sur l'armature urbaine française. L'étude s'est fondée sur l'analyse du tertiaire, et sur la hiérarchisation des centres urbains en fonction de la rareté des services; et il a fallu passer de l'échelle d'une région à celle du territoire national. C'est sur la base de cette étude qu'a été lancé en 1964 le programme des métropoles d'équilibre ${ }^{4}$.

21 Restait le problème des moyens pour réaliser ce projet. Le programme a été adopté par l'Assemblée nationale et sa réalisation confiée à la Datar. En réalité le problème était simple : on voulait que ces villes aient un rôle plus important du point de vue de la localisation du tertiaire supérieur, du tertiaire de haut niveau de rareté. Mais il manquait dans diverses régions la demande pour ce type de service ! La Datar a donc eu l'idée de profiter du développement industriel pour essayer de développer l'industrie dans les villes choisies tout en s'occupant de l'amélioration de l'espace urbain, surtout du centre-ville, pour pouvoir accueillir ce nouveau tertiaire.

Ce programme devait se réaliser sur le long terme, et l'on pensait à l'époque qu'il pouvait être mené à bien et que la France aurait des métropoles dans chacune de ses régions. En fait tout a été arrêté par la crise économique de 1974-75. La Datar a laissé de côté les programmes ambitieux qui envisageaient de vaincre les déséquilibres régionaux et s'est contenté de jouer le rôle de "pompier " de la crise dans un combat contre le chômage et toutes ses conséquences sociales, délaissant toute action stratégique au profit d'actions au coup par coup.

La Datar va ainsi perdre la seule possibilité de réaliser une véritable réorganisation du territoire français et sera d'ailleurs menacée de suppression en 1986 et 1988, jusqu'à ce qu'elle retrouve en 1990 une nouvelle finalité: celle de repenser les bases de l'aménagement du territoire en les adaptant aux nouvelles réalités de la société. C'est 
ainsi qu'est né le service " Prospective et Territoires ", dans lequel Michel Rochefort a travaillé, et où il a participé à la rédaction du livre Les territoires du futur ${ }^{5}$.

Le groupe de travail dont il a fait partie a commencé par une longue réflexion sur les relations entre structures économiques et aménagement du territoire dans l'optique nouvelle d'un monde néo-libéral, de la mondialisation et de la globalisation de l'économie.

La première conclusion assez évidente fut de mettre fin à l'habitude française de ne penser l'aménagement que dans les limites du territoire français, sans sortir de ses frontières. On a vite reconnu qu'il y avait une inadéquation fondamentale entre des programmes d'aménagement qui ne sortaient pas des frontières et l'ouverture de celles-ci au nouveau système de flux et d'interrelations économiques, conséquence du modèle néo-libéral à l'échelle européenne comme à l'échelle mondiale. Plutôt que de se soucier d'une hiérarchisation des villes, il fallait penser surtout aux grandes villes, à l'échelle européenne et à l'échelle mondiale, et tenter de les adapter à l'ouverture nécessaire aux marchés mondiaux et aux nouvelles échelles de fonctionnement de l'économie.

Le deuxième problème fut celui de l'interprétation néo-libérale de l'aménagement du territoire. Quelques-uns défendaient l'idée que les lois du marché étaient capables de réguler les déséquilibres territoriaux et qu'il fallait seulement laisser jouer le système des concurrences des entreprises et de leurs stratégies territoriales. Ainsi, le territoire, petit à petit s'adapterait au nouveau système économique.

27 La réflexion s'est donc rapidement portée sur la notion de déterritorialisation $d u$ système économique, et sur les nouvelles formes stratégiques qui structurent le territoire. Ce n'est plus la stratégie du secteur public qui organise les réseaux et les systèmes, mais les stratégies territoriales des grandes entreprises à l'échelle européenne et mondiale, qui s'occupent de trouver des lieux pour l'implantation de leurs entreprises, industrielles et tertiaires. Ces nouvelles stratégies des entreprises concourent au développement local et à de nouvelles interactions entre le secteur privé et le pouvoir local. Ceci renforce aussi la compétitivité entre les villes, chacune voulant obtenir des grandes entreprises le meilleur effet sur la croissance économique dans son territoire. Il y a donc une double concurrence : celle des grandes entreprises entre elles et celle des responsables des villes, chacun essayant d'obtenir plus d'avantages que l'autre.

On peut penser qu'en laissant ce processus fonctionner librement, des contradictions vont naître et compromettre le bon fonctionnement de l'économie néo-libérale, avec des blocages divers et des conflits sociaux. Ainsi apparaît l'idée que le néo-libéralisme économique doit être accompagné d'un néo-interventionnisme territorial de l'État.

La réflexion a également porté sur les mutations du système productif à la fin du fordisme et sa réorganisation fondée sur la flexibilité, les productions plus diversifiées et l'apparition d'un nouveau tertiaire d'appui : nouvelles entreprises intermédiaires pour accompagner l'innovation, tels les centres de recherche, de marketing, de rationalisation de la production, de publicité etc. Toutes ces entreprises ont tendance à se concentrer dans les plus grandes villes de France, et en particulier à Paris, en s'ouvrant aux entreprises européennes et mondiales. Ces mutations économiques ont donc des effets importants sur le système urbain. Elles se traduisent en effet par une nouvelle métropolisation, fondée sur les pôles qui travaillent à l'échelle internationale, et où se concentrent les nouvelles formes de la production industrielle plus attachées à 
la flexibilité et à ce nouveau tertiaire, soit quelques grandes villes françaises - les mieux localisées en fonction des communications et des facilités de relations avec l'extérieur, mais aussi les plus dynamiques - et d'abord Paris.

En contre-partie de cette évolution, les mutations économiques posent le problème des centres régionaux qui n'ont pas la perspective de devenir des «métropoles». Pour ceux-ci, il faut reprendre les critères de rareté des équipements, car compte tenu des transformations actuelles de la société, chaque centre régional ne peut posséder à lui seul tous les équipements. Il y a donc un choix qui peut s'opérer soit par le jeu de la libre-concurrence soit par l'intermédiaire de l'État. Sans revenir sur Paris et le désert français, une partie des espaces ruraux français connaissent une décadence de l'agriculture, une certaine diminution de la population. Faut-il alors intervenir pour pallier cette tendance?

31 Tout ceci conduit à envisager une nouvelle forme d'interventionnisme de l'État pour le réaménagement du territoire. Quelles pourraient en être les bases ? D'abord une leçon de modestie : les pouvoirs publics doivent renoncer à des programmes d'aménagement qui tenteraient d'inverser les tendances profondes de l'organisation de l'espace liées à la structure du système économique. Il faut accepter ce que les Français appellent les tendances lourdes, et en particulier le rôle prépondérant de Paris. Mais il faut prêter attention aux métropoles émergentes. C'est là que l'on peut tenter de renforcer le développement du nouveau tertiaire et l'interrelation entre les entrepreneurs privés et le secteur public pour que la France puisse avoir trois ou quatre métropoles de ce type. Ce nouvel interventionnisme doit aussi s'occuper des autres villes importantes, les grandes et les moyennes villes du territoire français. On peut imaginer, dans chaque région, un réseau de solidarité, d'interrelation entre trois ou quatre villes. Il ne s'agit plus de réseau hiérarchisé, mais de spécialisation des diverses grandes villes d'une même région dans un domaine précis (santé, enseignement universitaire ou autre) de façon à créer cette solidarité. Il faut surtout convaincre leurs habitants de la nécessité de créer cette solidarité et gagner les pouvoirs locaux à cette idée de spécialisation. On pourrait parler de "villes en réseau » et non plus de réseaux de villes, pour éviter la confusion avec le vieux concept de réseau urbain.

Reste le problème de la désertification des zones rurales. On a développé, à ce propos, la notion de "pays", petite zone de solidarité regroupant un certain nombre de communes rurales et une petite ville et qui peut devenir une sorte de micro-région de solidarité. Un des principaux facteurs de la désertification rurale réside dans les conditions de vie à la campagne. Faute de pouvoir doter toutes les communes des services nécessaires, ces unités de proximité, ces « pays ", pourraient ainsi contribuer à résoudre le problème, à travers la création d'un réseau de solidarité pour l'usage des services.

Les « villes en réseau » et les " pays » ouvrent une possibilité d'imaginer quel pourrait être un schéma du néo-interventionnisme dans le territoire français visant à diminuer les effets négatifs du fonctionnement néo-liberal de l'économie. Mais on doit se rappeler qu'il faut d'abord accepter les tendances lourdes, puis élaborer une sorte d'interventionnisme qui ne soit pas seulement sous la responsabilité de l'État, mais qui soit aussi assumé par les habitants. À chaque fois il faut parler de solidarité. La première chose à faire est donc de convaincre les habitants de l'intérêt de cette tentative d'améliorer le territoire pour qu'ils abandonnent l'idée d'espace fermé. Il faut passer de l'hostilité à la solidarité. 
décentralisation industrielle dans le contexte de l'État de Rio de Janeiro. Elle a parlé d'une configuration spatiale élaborée en grande partie par l'économie agroexportatrice et par les politiques de l'État centralisé durant la période qu'elle nous dit aller de G à G, de Getulio Vargas à Ernesto Geisel (des années trente jusqu'aux années quatre-vingt). La permanence de cette configuration territoriale pendant aussi longtemps, donne une image de stagnation qui peut être attribuée au rôle de Rio de Janeiro dans l'économie brésilienne et aussi dans la sphère politique, surtout après le déplacement de la capitale nationale à Brasilia et la fusion de deux États : Rio de Janeiro et Guanabara. Son hypothèse est que le contexte urbano-industriel de l'État de Rio de Janeiro est dominé par un profil ancien de centralisation et de concentration de la population, des activités et des ressources qui caractérise la ville de Rio de Janeiro en tant que métropole. Comment définir alors un ensemble de villes au sein de cette concentration métropolitaine qui compte aujourd'hui dix millions d'habitants, soit $80 \%$ de la population d'un État petit $(40000 \mathrm{~km} 2)$ par rapport à d'autres États de la fédération brésilienne, et où plus de $95 \%$ de la population est urbaine ? Le discours sur l'intégration de l'État de Rio de Janeiro constitue, selon l'auteur, un imaginaire de la population, mais il y a une séparation entre l'identité carioca (l'habitant de la ville de Rio de Janeiro) et celle des fluminenses (les autres habitants de l'État de Rio de Janeiro). Fany Davidovich a conclu son intervention en faisant allusion aux propositions de néointerventionisme présentées par Michel Rochefort qu'elle considère comme une nouvelle leçon qu'il nous apporte pour réfléchir à la situation de Rio de Janeiro. Rio, qui cherche une position dans l'économie globale va-t-elle pouvoir moderniser le territoire dont elle est la capitale ou va-t-elle laisser cet espace et opter pour un développement vertical.

Jorge Guilherme Francisconi est intervenu sur l'influence de Michel Rochefort dans l'aménagement du territoire et la politique urbaine au Brésil. En tant que secrétaireexécutif de la CNPU, il a invité Rochefort à participer aux projets de la commission, surtout dans le domaine théorique. Dans les années soixante-dix, la mission était de 
développer une politique urbaine globale et on ne pouvait plus se contenter d'utiliser les régions métropolitaines qui avaient été définies par un arrêté du Gouvernement (la fameuse loi $\mathrm{n}^{\circ} 14$ de 1974). Il fallait prendre en considération l'ensemble du système urbain en essayant de réduire le poids et la croissance de São Paulo. C'est à ce moment là que Michel Rochefort et Hélène Lamicq nous ont aidés à élaborer un cadre d'analyse et d'intervention sur l'urbanisation brésilienne qui a abouti à toute une série d'actions dans le domaine urbain au Brésil. Il s'agit en particulier du Programme des villes moyennes au Brésil (1978), partiellement financé par la Banque Mondiale. Ce groupe était extrêmement ambitieux : les réunions étaient riches du point de vue intellectuel et méthodologique : le programme des villes moyennes a été très bien accepté et a reçu un appui politique décisif des préfets.

40 Après une description intéressante de tous les problèmes politiques vécus pour l'élaboration de cette politique urbaine, Francisconi conclut: «C'est pour tout cela, et aussi pour d'autres apports que je n'ai pas eu le temps de raconter sur cette coopération entre Michel Rochefort et le gouvernement brésilien, que je suis ici aujourd'hui en hommage à un maître et à un ami. »

41 José Borzachiello da Silva a parcouru le chemin de la géographie brésilienne à partir de 1956, quand Michel Rochefort a entamé sa collaboration avec le Brésil. Ce qui me semble le plus intéressant dans sa contribution c'est l'analyse qu'il a faite des relations entre les autres géographies du monde et celle du Brésil : l'influence de la géographie française, mais aussi de la géographie anglo-saxonne, américaine et portugaise. Il nous a révélé toutes les polémiques et expliqué comment la géographie française est restée influente avec Rochefort au début, puis avec d'autres géographes français qui sont passés au Brésil et qui continuent à y venir et à influencer notre pensée en géographie physique, économique, culturelle etc. L'auteur a également souligné qu'au cours des vingt-cinq dernières années, Michel Rochefort a orienté 32 thèses de doctorat élaborées par des Brésiliens, et facilité la construction d'une pensée très attachée au professeur qui va du nord au sud du Brésil et qui consolide une certaine façon de comprendre l'urbain et la région dans notre pays.

Une table ronde coordonnée par Manoel Correia de Andrade et réunissant plusieurs géographes brésiliens, a insisté sur la nécessité de repenser ce qu'est le sousdéveloppement, et ce qu'il représente actuellement. Aujourd'hui il n'y a pas seulement des expulsions de la campagne, mais aussi des usines, des bureaux ou des mines.

De très nombreuses et intéressantes questions ont été posées lors de cette table ronde à propos de la signification de l'urbanisation et des villes dans les pays pauvres. Inez Costa Barboza a aussi replacé l'urbanisation dans le contexte de la production des inégalités et du rôle de l'État dans cette production et a proposé une série de thèmes de recherche pour mieux comprendre la nouvelle configuration territoriale de l'urbanisation brésilienne.

On peut donc voir, par ces résumés, la nature et la profondeur des discussions qui ont eu lieu à São Paulo. Ma condition de coordinatrice générale du séminaire ne m'a pas permis d'assister à tous les débats, et je n'ai évoqué ici que les sujets sur lesquels j'avais des notes personnelles et que j'ai eu la chance de pouvoir accompagner, mais toutes les contributions ont été de même importance. Elles font partie d'une collection de vidéos et de cassettes déposées au département de Géographie de l'université de São Paulo, qui constituent un matériau précieux sur l'hommage à Michel Rochefort, et sont ainsi un témoignage de son rôle dans les réflexions brésiliennes sur l'urbain et la région. 
45 notre maître, notre ami.

\section{NOTES}

1.Michel Rochefort, Redes e sistemas. Ensinando sobre o urbano e a Regiao, Hucitec, São Paulo, 1998

2.Michel Rochefort, L'organisation urbaine de l'Alsace, éd. Les Belles Lettres, Paris, 1960 3.Jean-François Gravier, Paris et le désert français, Flammarion, Paris, 1947

4.Voir dans cet ouvrage, Jeanine Cohen, « Métropoles d'équilibre : un géographe face au Politique » et Dominique Rivière « Michel Rochefort et l'aménagement ».

5.F. Ascher, L. Brams, M. Rochefort (et al.), Les territoires du futur, Paris, Éd. de l'aube, 1993.

\section{AUTEUR}

MARIA ADÉLIA APARECIDA DE SOUZA

Professeur de géographie humaine, université de São Paulo 